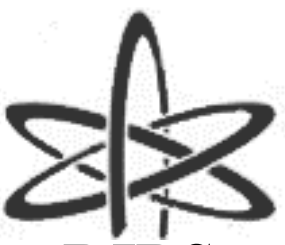

BJRS
BRAZILIAN JOURNAL

$\mathrm{OF}$

RADIATION SCIENCES

07-03B (2019) 01-09

\title{
Calibração de um sistema portátil para bioanálise de amostras de urina em situações de emergência
}

\author{
Erbe $^{a}$ A.L.D., Dantas ${ }^{a}$ A.L., Souza ${ }^{a}$ W.O., Dantas ${ }^{a}$ B.M. \\ ${ }^{a}$ Instituto de Radioproteção e Dosimetria, 22783-116, Av. Salvador Allende $s / n$ \\ amidegenhardt@gmail.com
}

\section{RESUMO}

A bioanálise in vitro é utilizada para monitoração interna da exposição em situações de rotina e emergência. Este trabalho descreve a calibração de um sistema de deteção portátil para medição de radionuclídeos em amostras de urina utilizando-se fonte líquida padrão de ${ }^{152} \mathrm{Eu}$ na geometria de $1 \mathrm{~L}$. A sensibilidade do sistema foi avaliada com base na dose efetiva mínima detetável para ${ }^{103} \mathrm{Ru},{ }^{106} \mathrm{Ru},{ }^{134} \mathrm{Cs},{ }^{137} \mathrm{Cs}$ e ${ }^{60} \mathrm{Co}$, utilizando-se o software AIDE e supondo o cenário de incorporação. Exceto para o ${ }^{106} \mathrm{Ru}$, o sistema apresentou sensibilidade suficiente para medir atividades decorrentes de incorporações que resultem em doses efetivas abaixo de 1 mSv.

Keywords: monitoração interna, bioanálise de urina, resposta a emergência.

\begin{abstract}
In vitro bioassay is used for internal monitoring of the exposure in routine and emergency situations. This work describes de calibration of a portable detection system for the measurement of radionuclides in urine samples using a liquid standard source of ${ }^{152} \mathrm{Eu}$ in $1 \mathrm{~L}$ geometry. System sensitivity was evaluated based on the minimum detectable effective dose for ${ }^{103} \mathrm{Ru},{ }^{106} \mathrm{Ru},{ }^{134} \mathrm{Cs},{ }^{137} \mathrm{Cs}$ e ${ }^{60} \mathrm{Co}$ using AIDE software and considering a scenario of intake by inhalation. Except for ${ }^{106} \mathrm{Ru}$, the system presented enough sensitivity to measure activities excreted as a result of intakes which result in effective doses below $1 \mathrm{mSv}$.
\end{abstract}

Keywords: internal monitoring, urine bioassay, prompt response to emergency. 


\section{INTRODUÇÃO}

Materiais radiativos são amplamente utilizados na indústria, medicina, pesquisa e ensino, e até mesmo em produtos à venda diretamente para o público em geral [1]. Eles são utilizados para radiografia industrial, esterilização de materiais, radioterapia, medicina nuclear e detetores de fumaça. Fontes que tenham sido roubadas ou perdidas em laboratórios, indústrias ou no seu transporte, podem dar origem a acidentes radiológicos [1].

Em situações de acidentes radiológicos e nucleares, existe o risco de incorporação de radionuclídeos por indivíduos da população, tornando as monitorações interna e externa elementos essenciais em uma resposta a emergência [2]. As ações de remediação nestes casos inclui monitoração ambiental e individual, e permite a classificação do acidente, direciona decisões e ações de intervenção com base em níveis operacionais de intervenção (OIL), previne a disseminação da contaminação, auxilia na obtenção de informações para segurança dos trabalhadores da emergência e sobre os perigos resultantes do acidente, determina a extensão e duração dos perigos ocasionados, e confirma eficácia das medidas remediadoras e procedimentos de descontaminação [3].

A monitoração interna em situações de emergência difere da monitoração ocupacional de rotina, pois requer resultados rápidos e capacidade de atender locais distantes e de difícil acesso.

Este trabalho descreve a calibração e avaliação da sensibilidade de um sistema portátil de monitoração interna disponível no Laboratório de Monitoração in vivo do Instituto de Radioproteção e Dosimetria (LABMIV-IRD), destinado à medição de emissores gama de alta energia em amostras de urina para utilização em missões de pronta resposta a situações de emergência. 


\section{MATERIAIS E METODOS}

\subsection{Materiais utilizados}

Os materiais utilizados neste trabalho estão disponíveis no IRD, e consistem no seguinte:

- Detetor cintilador $\mathrm{NaI}(\mathrm{Tl}) 3 \times 3$ da marca CANBERRA ${ }^{\circledR}$, modelo 802, com a Base Universal para tubo MCA modelo Osprey ${ }^{\mathrm{TM}}$;

- Frasco de polietileno de $1 \mathrm{~L}$ contendo solução-padrão de ${ }^{152} \mathrm{Eu}$ diluída em meio ácido (HNO3 a 0,1 mol.L ${ }^{-1}$ ), fornecida pelo Laboratório Nacional de Metrologia das Radiações Ionizantes (LNMRI/ IRD), com atividade de 8448 Bq em 13/07/2017;

- Frasco de polietileno de 1L preenchido com água destilada para contagem da radiação de fundo;

- Fonte puntiforme de ${ }^{152} \mathrm{Eu}$ com atividade de $121730 \mathrm{~Bq}$ em 28/12/2000, para calibração em Canal x Energia;

- Software AIDE, versão 6.0 disponível no LABMIV/IRD, para cálculos de estimativa de dose.

\subsection{Metodologia}

Primeiramente foi realizada a calibração em "canal vs energia" utilizando-se uma fonte puntiforme de ${ }^{152} \mathrm{Eu}$. Este tipo de calibração relaciona os valores das energias fótons mais intensos do ${ }^{152} \mathrm{Eu}(121,8 \mathrm{keV}, 244,7 \mathrm{keV}, 344,3 \mathrm{keV}, 778,9 \mathrm{keV}, 1112,1 \mathrm{keV}$ e $1408,0 \mathrm{keV})$ com os respectivos canais dos fotopicos.

Em seguida, foi realizada a calibração em eficiência do sistema de deteção consiste na obtenção de uma curva "Eficiência x Energia" para a geometria específica de interesse, neste caso, Frasco de $1 \mathrm{~L}$.

Posteriormente são calculadas as atividades mínimas detectáveis e doses efetivas mínimas detectáveis para radionuclídeos de interesse na geometria de medição.

Cinco medidas consecutivas de 600 segundos foram realizadas com o detetor colimado (distância do topo do frasco ao detetor $=2,5 \mathrm{~cm}$ ). As contagens foram registradas em cinco regiões 
de interesse correspondentes aos fótons de 121,78 keV, 244,7 keV, 344,3 keV, 778,9 e 964,10 keV do ${ }^{152} \mathrm{Eu}$. A eficiência de deteção em cada ROI foi calculada da seguinte forma:

$$
\mathrm{Ef}=\frac{(\mathrm{C} / \mathrm{T})}{(\mathrm{A} \times \operatorname{Ig})}
$$

onde Ef é a eficiência de deteção no ROI do fóton de interesse; $\mathrm{C}$ é o total de contagens no ROI; T é o tempo de contagem (s); A é a atividade da fonte padrão de ${ }^{152} \mathrm{Eu}(\mathrm{Bq})$ e $\operatorname{Ig}$ é a intensidade de emissão gama na energia medida.

Para a avaliação a sensibilidade da técnica, foram selecionados cinco radionuclídeos de interesse para monitoração interna em caso de um acidente nuclear: ${ }^{103} \mathrm{Ru},{ }^{106} \mathrm{Ru},{ }^{134} \mathrm{Cs},{ }^{137} \mathrm{Cs}$ e ${ }^{60}$ Co. A partir da curva "Eficiência x Energia", foram calculadas as eficiências de deteção para as respectivas energias gama e as Atividades Mínimas Detectáveis (MDA) para cada radionuclídeo.

$$
\mathrm{AMD}_{\mathrm{Bq}}=\frac{4,65 \cdot \sqrt{\mathrm{N}}}{\mathrm{T} \times \mathrm{Ef} \times \mathrm{Ig}}
$$

onde AMD é a Atividade Mínima Detetável (Bq); N é o total de contagens do BG na ROI em 300 segundos; Ef é a eficiência de deteção (cps/dps), T é o tempo de contagem e Ig é a intensidade de emissão gama na energia medida.

Seguindo as recomendações da publicação AIEA RSG 1-2 (AIEA, 1999), o software AIDE foi utilizado para calcular a atividade presente na urina de 24 horas resultante de inalação única de $1 \mathrm{~Bq}$ de ${ }^{103} \mathrm{Ru},{ }^{106} \mathrm{Ru}$ e ${ }^{60} \mathrm{Co}$ na forma de particulados com AMAD $=1 \mu \mathrm{m}$ e Classe de solubilidade no trato respiratório tipo $\mathrm{M}$.

$\mathrm{O}$ cenário utilizado para o ${ }^{134} \mathrm{Cs}$ e ${ }^{137} \mathrm{Cs}$ foi inalação única, $\mathrm{AMAD}=1 \mu \mathrm{m}$, absorção tipo $\mathrm{F}$. Com base nas frações de excreção " $\mathrm{m}(\mathrm{t})$ " fornecidas pelo software, foi calculada a Incorporação Mínima Detetável para esses radionuclídeos, supondo que a atividade medida na urina é igual à AMD da técnica.

$$
\mathrm{IMD}_{\mathrm{Bq}}=\frac{\mathrm{AMD}}{\mathrm{m}(\mathrm{t})_{\text {inalação }}}
$$


onde AMD é a atividade mínima detetável $(\mathrm{Bq})$ e $\mathrm{m}(\mathrm{t})$ é a fração de excreção de urina $(\mathrm{Bq} / \mathrm{Bq})$.

Usando o valor de IMD e o coeficiente de dose dado pelo software AIDE, calculou-se a dose efetiva detetável mínima para os radionuclídeos de interesse.

$$
\mathrm{DEMD}=\mathrm{IMD} * \mathrm{e}(50)
$$

Onde DEMD é a dose efetiva comprometida mínima detetável (mSv) e e(50) é o fator de dose efetiva comprometida $(\mathrm{mSv} / \mathrm{Bq})$.

\section{RESULTADOS E DISCUSSÃO}

A figura 1 apresenta a curva de calibração "Canal vs energia" obtida com fonte padrão de ${ }^{152}$ Eu. O ajuste foi realizado pelo software do multicanal.

Figura 1:Curva de calibração "Canal vs energia" do detetorr cintilador NaI(Tl)3x3 CANBERRA 802.

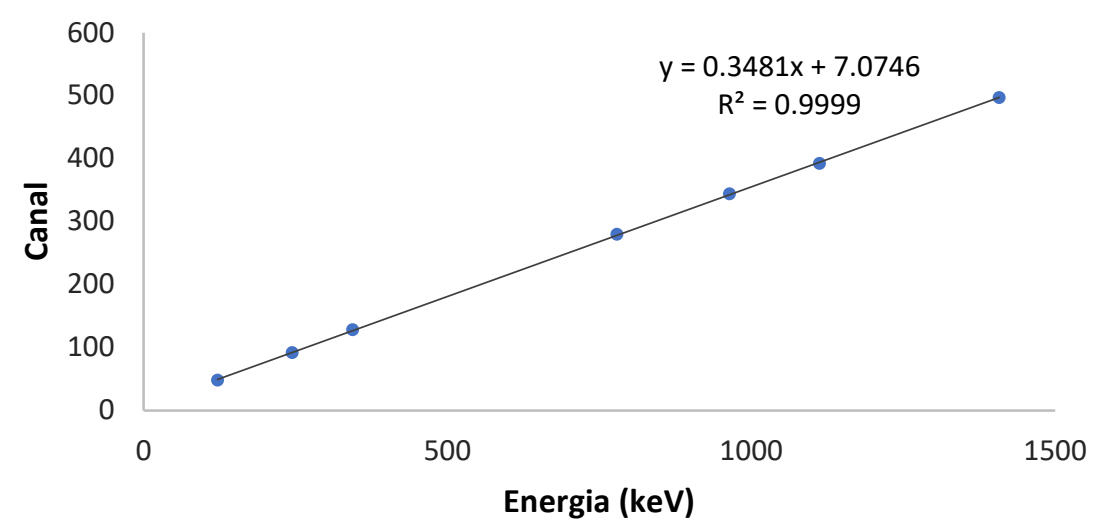

A geometria de medição do frasco de 1L está demonstrado na figura 2. 
Figura 2 : Geometria de medição do amostra líquida em frasco de $1 L$ com detetor NaI(Tl) $3 x 3$.

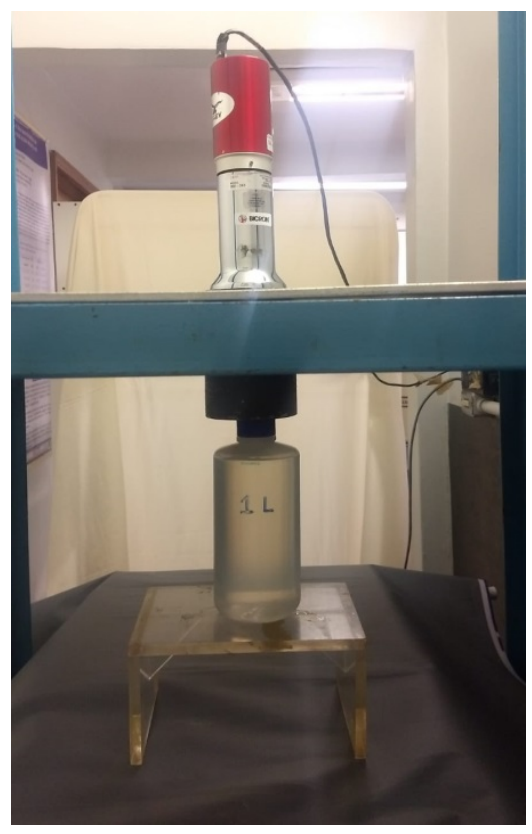

Foram calculadas as eficiências de detecção para as 5 energias de emissão gama do ${ }^{152} \mathrm{Eu}$. A curva de eficiência vs. energia é apresentada na figura 3.

Figura 3 :Curva de eficiência vs energia do detetorr NaI(Tl)3x3 colimado para a geometria de frasco de $1 L$.

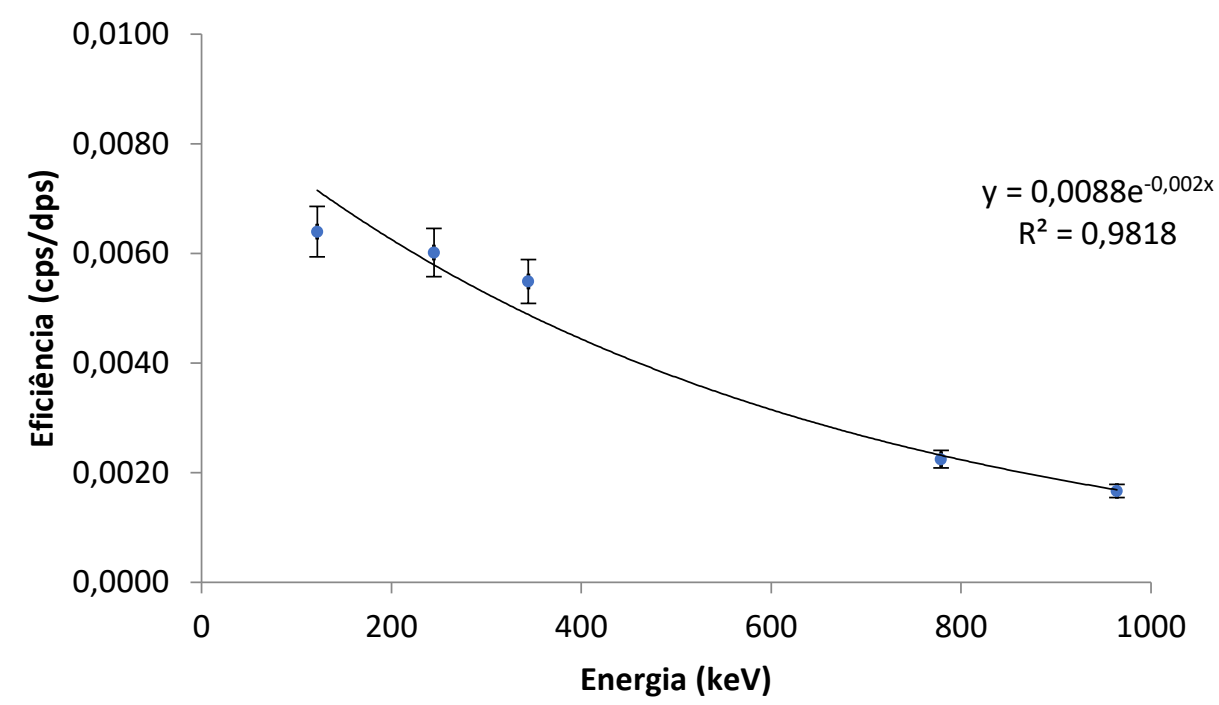


A tabela 1 mostra as incertezas respectivas à eficiência em cada energia das ROI, que foram estimadas considerando a incerteza da atividade da fonte, cerca de 3,6 \%, e a incerteza da contagem líquida.

Tabela 1: Incertezas da eficiência em cada RDI

\begin{tabular}{cccc}
\hline Energia & Eficiência & Incerteza & $\%$ \\
\hline 121,78 & 0,0064 & $\pm 0,00023$ & 3,60 \\
244,7 & 0,0060 & $\pm 0,00022$ & 3,66 \\
344,28 & 0,0055 & $\pm 0,00020$ & 3,64 \\
778,9 & 0,0022 & $\pm 0,00008$ & 3,56 \\
964,1 & 0,0017 & $\pm 0,00006$ & 3,60 \\
\hline
\end{tabular}

A tabela 2 relaciona os radionuclídeos de interesse e suas respectivas energias de emissão gama.

Tabela 2: Energias de emissão gama dos radionuclídeos de interesse.

\begin{tabular}{cc}
\hline Radionuclideo & Energia (kev) \\
\hline${ }^{106} \mathrm{Ru}$ & 155,03 \\
${ }^{103} \mathrm{Ru}$ & 497,1 \\
${ }^{134} \mathrm{Cs}$ & 604,7 \\
${ }^{137} \mathrm{Cs}$ & 661,6 \\
${ }^{60} \mathrm{Co}$ & 1173,3 \\
\hline
\end{tabular}

As AMDs para cada radionuclídeo de interesse se encontram na tabela 3. 
Tabela 3: AMD para cada radionuclídeo de interesse.

\begin{tabular}{cc}
\hline Radionuclideo & AMD $(\mathrm{Bq})$ \\
\hline${ }^{106} \mathrm{Ru}$ & 1810 \\
${ }^{103} \mathrm{Ru}$ & 475 \\
${ }^{134} \mathrm{Cs}$ & 484 \\
${ }^{137} \mathrm{Cs}$ & 535 \\
${ }^{60} \mathrm{Co}$ & 819 \\
\hline
\end{tabular}

$\mathrm{O}{ }^{106} \mathrm{Ru}$ apresentou o mais elevado valor de AMD. Isso pode ser explicado pela sua baixa intensidade de emissão gama, cerca de $15 \%$, e pela elevada radiação de fundo na sua RDI, em relação aos demais radionuclídeos avaliados. Isto torna necessária a presença de maior atividade de ${ }^{106} \mathrm{Ru}$ na amostra de maneira que seja possível sua identificação e quantificação.

A tabela 4 fornece os valores de $\mathrm{m}(\mathrm{t})$, obtidos com auxílio do Software AIDE, de acordo com o cenário de incorporação simulado, IMD calculado com base no valor de AMD para cada radionuclídeo de interesse, e(50) (também fornecido pelo AIDE de acordo com o cenário de incorporação) e a DEMD calculada com base na IMD e e(50) para a geometria de medição.

Tabela 4 : Valores de m(t), IMD, e(50) e DEMD para cada radionuclídeo de interesse.

\begin{tabular}{ccccc}
\hline Radionuclídeo & $\mathrm{m}(\mathrm{t})(\mathrm{Bq} / \mathrm{Bq})$ & $\mathrm{IMD}(\mathrm{Bq})$ & $\mathrm{e}(50)(\mathrm{mSv} / \mathrm{Bq})$ & $\mathrm{DEMD}(\mathrm{mSv})$ \\
\hline${ }^{106} \mathrm{Ru}$ & $4,02 \times 10^{-3}$ & $45,55 \times 10^{5}$ & $2,60 \times 10^{-5}$ & 11,7 \\
${ }^{103} \mathrm{Ru}$ & $3,96 \times 10^{-3}$ & $88,44 \times 10^{3}$ & $2,26 \times 10^{-6}$ & 0,3 \\
${ }^{134} \mathrm{Cs}$ & $4,05 \times 10^{-3}$ & $81,19 \times 10^{3}$ & $6,80 \times 10^{-6}$ & 0,8 \\
${ }^{137} \mathrm{Cs}$ & $5,71 \times 10^{-3}$ & $62,61 \times 10^{3}$ & $4,80 \times 10^{-6}$ & 0,4 \\
${ }^{60} \mathrm{Co}$ & $7,75 \times 10^{-3}$ & $10,97 \times 10^{4}$ & $9,15 \times 10^{-6}$ & 1,0 \\
\hline
\end{tabular}

Para os radionuclídeos ${ }^{103} \mathrm{Ru},{ }^{134} \mathrm{Cs},{ }^{137} \mathrm{Cs}$ e ${ }^{60} \mathrm{Co}$, o sistema se mostrou sensível para avaliação de incorporação que resultem em doses efetivas comprometidas abaixo ou igual a $1 \mathrm{mSv}$. No caso do ${ }^{106} \mathrm{Ru}$, o valor de DEMD foi de $11,8 \mathrm{mSv}$. Isso pode ser relacionado às características de emissão de fótons, conforme discutido anteriormente. 


\section{CONCLUSÕES}

Com base nos resultados obtidos é possível concluir que o sistema de deteção avaliado possui sensibilidade suficiente para avaliação de incorporações que resultem em doses efetivas comprometidas abaixo ou igual $1 \mathrm{mSv}$ para os radionuclídeos ${ }^{103} \mathrm{Ru},{ }^{134} \mathrm{Cs},{ }^{137} \mathrm{Cs}$ e ${ }^{60} \mathrm{Co}$. Para o radionuclídeo ${ }^{106} \mathrm{Ru}$ a dose efetiva mínima detetável alcançada, nas condições de geometria de deteção e tempo de contagem, foi de 11,8 mSv. Este resultado, entretanto, não inviabiliza a utilização da técnica para avaliação de incorporações em situações de acidente.

\section{REFERÊNCIAS}

[1] IAEA - INTERNATIONAL ATOMIC ENERGY AGENCY. Generic Procedures for Assessment and Response during a Radiological Emergency, IAEA-TECDOC-1162, Vienna (2000).

[2] LI. C, ANSARI. A, ETHERINGTON. G, JOURDAIN. JR, KUKHTA. B, KURIHARA. O, LOPEZ. MA, MÉNÉTRIER. F, ALVES DOS REIS A., SOLOMON. S, ZHANG. J, CARR. Z, Managing Internal Radiation Contamination Following an Emergency: Identification of Gaps and Priorities. Radiat Prot Dosimetry. Sep;171(1):78-84, 2016.

[3] IAEA - INTERNATIONAL ATOMIC ENERGY AGENCY. Assessment of Occupational Exposure Due to Intakes of Radionuclides. Safety Report Series No. RS-G-1.2, Vienna, 1999. 